\title{
Propagator with positive cosmological constant in the 3D Euclidean quantum gravity toy model
}

\author{
William E Bunting ${ }^{1}$ and Carlo Rovelli ${ }^{2}$ \\ ${ }^{1}$ California Institute of Technology, Pasadena, CA 91125, USA \\ ${ }^{2}$ Aix Marseille Université, CNRS, CPT, UMR 7332, 13288 Marseille, France \\ E-mail: wbunting@ caltech.edu
}

Received 2 May 2014, revised 3 June 2014

Accepted for publication 16 June 2014

Published 22 July 2014

\begin{abstract}
We study the propagator on a single tetrahedron in a three-dimensional toy model of quantum gravity with positive cosmological constant. The cosmological constant is included in the model via q-deformation of the spatial symmetry algebra, that is, we use the Turaev-Viro amplitude. The expected repulsive effect of dark energy is recovered in numerical and analytic calculations of the propagator at large scales comparable to the infrared cutoff. However, due to the simplicity of the model, we do not obtain the exact Newton limit of the propagator. This is a first step toward the similar calculation in the full $3+1$ dimensional theory with larger numbers of simplicies.
\end{abstract}

Keywords: 3D toy model, quantum gravity, cosmological constant

\section{Introduction}

This paper extends work [1-3] evaluating quantum gravity two-point functions on a single tetrahedron in three Euclidean dimensions to the case with positive cosmological constant. At long distance scales, the two point function for a quantum field theory gives the Newton force associated with that theory's gauge boson. In our toy model, since we only have one tetrahedron, and we peak our state around an equilateral configuration of that tetrahedron, we do not expect to reproduce the exact Newton limit of 3D gravity with a cosmological constant. However, we still do find an asymptotically repulsive force associated with dark energy.

We take the inclusion of the cosmological constant to correspond to a deformation of the $S U$ (2) spatial rotation symmetry. This quantum gravity toy model is known as the qdeformed Ponzano-Regge model or the Turaev-Viro model. We expect two key differences in the propagator from the Ponzano-Regge model. First, in the case where the tetrahedra is large compared with the infrared cutoff imposed by the cosmological constant, the sums will 
be cut off by the deformation and not the triangular inequalities. This feature of the quantum algebra is essential in four dimensions where we cannot escape so-called 'bubble' configurations of 2-complexes, and without an infrared cutoff these transition amplitudes would diverge. Second, in the case where the size of the tetrahedron is smaller than the cutoff, the modification will simply affect the asymptotics of the two point function via the addition of a volume term to the Regge action.

In this paper we first compute the Turaev-Viro propagator numerically in section 2 . Then we compare the numerical computations with an analytic calculation of the propagator asymptotics in section 3. These two calculations are found to match strongly in an easily computable regime, that is, for an unrealistically large cosmological constant. However, the agreement is expected to persist as the cosmological constant is taken smaller.

\section{Method and results}

This paper studies the correlator between two edges on a single tetrahedron. We fix four edges around the tetrahedron to $j_{0}$, which can be thought of as a time. Thus, $j_{1}$ and $j_{2}$ are lengths on two different time slices of the spacetime, see figure 1 . We will study the modulus of the propagator $|\mathcal{P}|$ as a function of the distance $j_{0}$ and the infrared cutoff $j_{\max }$.

Because in the 3D case all of the calculations can be done in a gauge where they all give zero, we clearly have an issue of gauge choice. We follow Speziale by picking a Coulomblike gauge where the field operators have non-trivial projections along a bone (edge of a triangle in the triangulation) [1]. This choice will produce a calculation similar to the one that can be done in the 4D spinfoam model [4]. We choose our operator notation to be different from Speziale, but in line with more recent work in quantum gravity correlation functions $[5,6]$. The two point function is:

$$
\mathcal{P}_{n m}^{a b c d}:=\frac{\left\langle W\left|\mathcal{P}_{n}^{a b} \mathcal{P}_{m}^{c d}\right| \psi_{\Sigma}\right\rangle_{q}}{\left\langle W \mid \psi_{\Sigma}\right\rangle_{q}}
$$

where instead of the usual metric field insertions we have the operators perturbed around flat space.

$$
\begin{aligned}
\mathcal{P}_{n}^{a b}|s\rangle & :=l_{a, n}^{\mu} l_{b, n}^{\nu} h_{\mu \nu}(x)|s\rangle \\
& =l_{a, n}^{\mu} l_{b, n}^{\nu}\left(g_{\mu \nu}(x)-\delta_{\mu \nu}\right)|s\rangle \\
& =\left(l_{p}^{2} G_{n}^{a b}-l_{p}^{2} C_{q}^{2}\left(j_{0}\right)\right)|s\rangle .
\end{aligned}
$$

Above $G_{n}^{a b}:=\vec{L}_{n}^{a} \cdot \vec{L}_{n}^{b}$ are the Penrose operators acting on links that go between nodes $a$ and $n$, and $b$ and $n$ in the spin network state $|s\rangle, C_{q}$ is the $S U_{q}(2)$ casimir defined as $C_{q}(j):=\sqrt{[j][j+1]+1 / 4}, W$ is the Turaev-Viro transition amplitude and $\psi_{\Sigma}$ is a boundary state peaked on an equilateral tetrahedron. For a tetrahedron, the spin network state is also a tetrahedron dual to the original one where there are nodes on all the faces. We would like to choose the node labels such that we are calculating the correlator between the two edges labeled $j_{1}$ and $j_{2}$. For instance, we could label the four nodes such that face 1 opposes face 3 and face 2 opposes face 4 . Then we would be interested in $\mathcal{P}_{34}^{1122}$. We calculate this by evaluating equation (1) with the q-deformed state and transition amplitude obtained from summing over amplitudes for different configurations of the geometry, i.e.: 


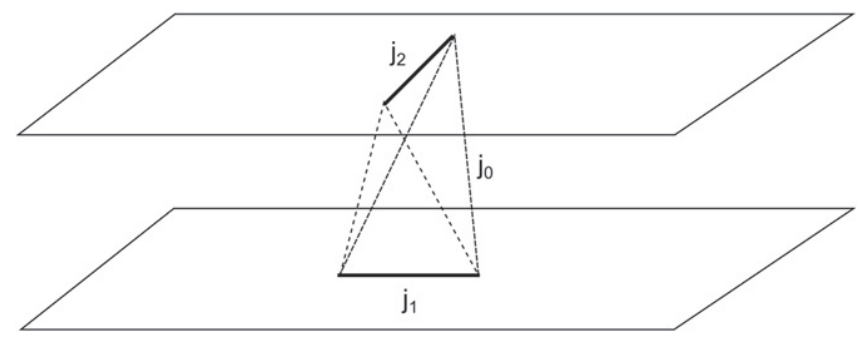

Figure 1. Depiction of the tetrahedron state used in this paper. The four edges of the tetrahedron that go between the boundary slices are fixed to a value of $j_{0}$ and can be thought to correspond to the time. We then study the two point function between the length operators acting on each of the two edges on the slices.

$$
\begin{aligned}
\mathcal{P}_{34}^{1122}= & \frac{1}{j_{0}^{4} l_{p}^{4} \mathcal{N}} \sum_{j_{1}, j_{2}}^{2 j_{0}} W_{q}\left(j_{1}, j_{2} ; j_{0}\right) \psi_{\Sigma}^{q}\left(j_{1}, j_{2} ; j_{\max }\right) l_{p}^{4}\left(C_{q}^{2}\left(j_{1}\right)\right. \\
& \left.-C_{q}^{2}\left(j_{0}\right)\right)\left(C_{q}^{2}\left(j_{2}\right)-C_{q}^{2}\left(j_{0}\right)\right),
\end{aligned}
$$

where $\mathcal{N}$ is a normalization factor, and we have from the Turaev-Viro model that the transition amplitude for a single tetrahedron is just a quantum $6 j$-symbol:

$$
W_{q}\left(j_{1}, j_{2} ; j_{0}\right)=\left\{\begin{array}{ccc}
j_{1} & j_{0} & j_{0} \\
j_{2} & j_{0} & j_{0}
\end{array}\right\}
$$

we take the deformation parameter to be a root of unity $q=\exp (i \sqrt{\Lambda} \hbar G)$ where $\Lambda$ is the cosmological constant that fixes the infrared cutoff $j_{\max }$ as $\sqrt{\Lambda}=\pi /\left(2 j_{\max }+1\right)$ [7]. The boundary state is taken to be:

$$
\psi_{\Sigma}^{q}=\frac{1}{N} \exp \left(-\frac{\alpha}{2} \sum_{i}\left(j_{i}-j_{0}\right)^{2}+i \theta \sum_{i}\left(j_{i}+\frac{1}{2}\right)-i \frac{\sqrt{\Lambda} j_{0}^{2}}{12 \sqrt{2}} \sum_{i}\left(j_{i}-j_{0}\right)\right) .
$$

We pick a Gaussian state so as to peak the geometry on that of a (spherical) tetrahedron, see Speziale [1]. One might want to change the state to something other than a Gaussian, like a Bessel state, but the leading order behavior is the same [2,3] and so we stick with a Gaussian for simplicity. Note that we need to pick the factor $\alpha$ in such a way as to enforce the vanishing of relative uncertainties of $j$ and $\theta$ in the semi-classical limit. The factor with $\Lambda$ in this state is included so that the asymptotics do not have oscillatory terms in the limit as $j_{0} \rightarrow \infty$ that come from the addition of the cosmological constant term to the Regge action. Numerical evaluation of equation (2) gives the main result of the paper, see figure 2 . Here we can see that at small scales $j_{0} \approx 1$ we have the same quantum deviations as Speziale from the Newtonian limit. In the range where we are not too close to the cutoff and have not too small lengths, i.e. $1 \ll j_{0} \ll j_{\max } / 2$, we have behavior similar to the Newtonian limit without cosmological constant $|\mathcal{P}| \approx 3 / 2 j_{0}$. Lastly, when the spins get close to the infrared cutoff we have a repulsion representative of the repulsive force of dark energy.

\section{Discussion and analysis}

We would like to analyze analytically the asymptotics of this toy model to see if we can match the numerical result. The asymptotics of $\{6 j\}_{q}$ symbols are given by a cosine of the 


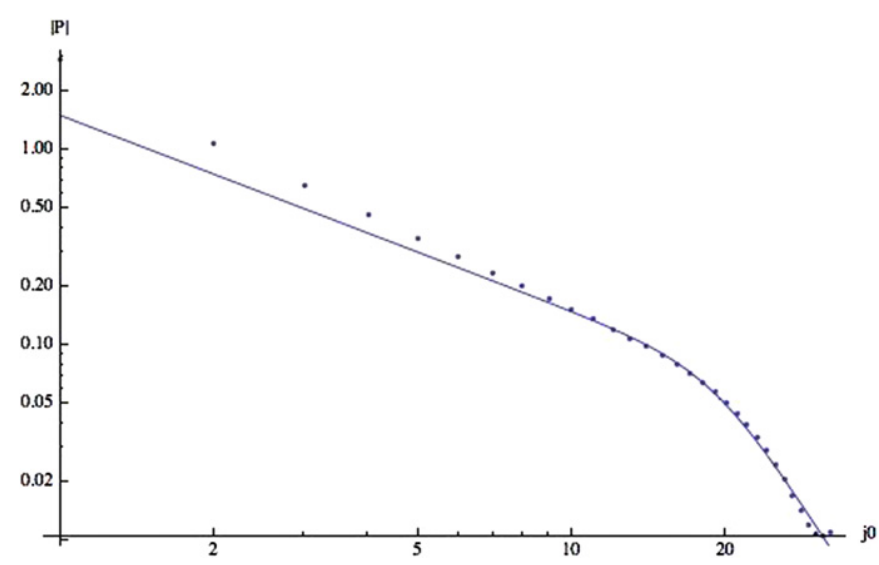

Figure 2. Plot of the q-deformed propagator calculated for $j_{\max }=65$ and various values of $j_{0}$. The asymptotic behavior is clearly modified by the presence of the cosmological constant via a repulsion at large distances. This repulsion is well captured in the analytic calculation of the curve in the figure. Additionally, in the intermediate regime where $1 \ll j_{0} \ll j_{\max } / 2$, we have agreement with the Speziale asymptotics, i.e. $|\mathcal{P}| \sim 3 / 2 j_{0}$.

Regge action with a volume term [8, 9].

$$
\{6 j\}_{q} \sim \frac{2 \pi \cos \left(S_{R}\left[j_{e}\right]-\sqrt{\Lambda} \operatorname{Vol}\left(\tau_{S}\right)+\frac{\pi}{4}\right)}{r^{3 / 2} G^{1 / 4}}
$$

The volume term is the volume of a spherical tetrahedron. We will approximate this as a flat tetrahedron and expand the volume in $\delta j_{1}$ and $\delta j_{2}$ as:

$$
\begin{gathered}
\operatorname{Vol}\left(\tau_{S}\right) \approx \operatorname{Vol}\left(\tau_{E}\right) \approx \frac{\left(1+2 j_{0}\right)^{3}}{48 \sqrt{2}}+\frac{\left(1+2 j_{0}\right)^{2}}{48 \sqrt{2}}\left(\delta j_{1}+\delta j_{2}\right) \\
-\frac{7\left(1+2 j_{0}\right)}{96 \sqrt{2}}\left(\delta j_{1}^{2}+\delta j_{2}^{2}\right)-\frac{\left(1+2 j_{0}\right) \delta j_{1} \delta j_{2}}{48 \sqrt{2}} .
\end{gathered}
$$

The factor of $2 \pi / r^{3 / 2} G^{1 / 4}$ is approximately independent of $j_{1}$ and $j_{2}$, therefore it will cancel with the same factor in the normalization. Also in the large spin limit the difference of the Casimir operators will go like $C^{2}\left(j_{1}\right)-C^{2}\left(j_{0}\right) \sim 2 j_{0} \delta j_{1}$. We then arrive at an asymptotic formula for the propagator:

$$
\begin{aligned}
|\mathcal{P}| & \sim \frac{4}{j_{0}^{2} \mathcal{N}} \sum_{j_{1}, j_{2}}^{2 j_{0}} \delta j_{1} \delta j_{2} \exp \left[-i S_{R}\left[j_{e}\right]+\sqrt{\Lambda} i \operatorname{Vol}\left(\tau_{E}\right)\right. \\
& \left.-i \frac{\pi}{4}-\frac{\alpha}{2} \sum_{i} \delta j_{i}^{2}+i \theta \sum_{i}\left(j_{i}+\frac{1}{2}\right)-\frac{i \sqrt{\Lambda} j_{0}^{2}}{12 \sqrt{2}} \sum_{i} \delta j_{i}\right]
\end{aligned}
$$

where we have omitted the other exponential term that is rapidly oscillating in $j_{1}$ and $j_{2}$. Expanding action and the volume terms around the equilateral configuration, and canceling factors independent of $j_{1}$ and $j_{2}$ with the same ones in the normalization, we obtain: 


$$
\begin{aligned}
|\mathcal{P}| & \sim \frac{4}{j_{0}^{2} \mathcal{N}} \sum_{j_{1}, j_{2}}^{2 j_{0}} \delta j_{1} \delta j_{2} \exp \left[-\frac{i \sqrt{\Lambda}}{2}\left(\frac{7 j_{0}}{24 \sqrt{2}}\left(\delta j_{1}^{2}+\delta j_{2}^{2}\right)+\frac{j_{0} \delta j_{1} \delta j_{2}}{12 \sqrt{2}}\right)\right. \\
& \left.-\frac{i}{2} \sum_{i, k} G_{i k} \delta j_{i} \delta j_{k}-\frac{\alpha}{2} \sum_{i=1}^{2} \delta j_{i}^{2}\right],
\end{aligned}
$$

where $G_{i k}=\partial \theta_{i} /\left.\partial j_{k}\right|_{j_{e}=j_{0}}$ is the matrix of derivatives of the dihedral angles with respect to the edge lengths evaluated for an equilateral tetrahedron. Passing to continuous variables $z=\delta j_{1}$ and $d z=d j_{1}$ we obtain the Gaussian integral:

$$
\mathcal{P} \sim \frac{4}{j_{0}^{2} \mathcal{N}} \int d^{2} z z_{1} z_{2} \exp \left[-\frac{1}{2} z_{i} A_{i k} z_{k}\right]=\frac{4}{j_{0}^{2}}\left(A^{-1}\right)_{12},
$$

where $A$ is the matrix of coefficients of $\delta j_{i} \delta j_{k}$ :

$$
A_{i k}=\frac{4}{3 j_{0}}\left(\begin{array}{cc}
1+i \frac{\sqrt{2}}{4} & i \frac{3 \sqrt{2}}{4} \\
i \frac{3 \sqrt{2}}{4} & 1+i \frac{\sqrt{2}}{4}
\end{array}\right)+\frac{i \sqrt{\Lambda}\left(j_{0}+1 / 2\right)}{24 \sqrt{2}}\left(\begin{array}{ll}
7 & 1 \\
1 & 7
\end{array}\right)
$$

where we have set $\alpha=4 / 3 j_{0}$ which is compatible with the requirement that the state be peaked on the intrinsic and extrinsic geometry [1]. From this, we have the full expression for the asymptotics of the propagator modulus:

$$
|\mathcal{P}| \sim \frac{4 \sqrt{6}\left(96+J_{\Lambda}\right)^{2}}{j_{0}} \frac{1}{\sqrt{2^{17} \cdot 3+J_{\Lambda}\left(2^{13}+J_{\Lambda}\left(128+J_{\Lambda}\left(32+3 J_{\Lambda}\right)\right)\right)}}
$$

where $J_{\Lambda}:=j_{0}\left(1+2 j_{0}\right) \sqrt{\Lambda}$. The plot of the analytic expression versus the numerical calculation is shown in figure 2 , where their agreement is clear. If we expand this expression about $\Lambda=0$ it becomes more clear that a repulsion is the dominating correction to the Speziale result.

$$
|\mathcal{P}| \sim \frac{3}{2 j_{0}}-\frac{1}{1536}\left(j_{0}+1 / 2\right)^{2} j_{0} \Lambda+O\left(\Lambda^{3 / 2}\right)
$$

We obtain an expression with a term proportional to the cosmological constant times the volume of the tetrahedron. While this differs from what we might expect from the Newton law, we should remember that this is a simplified model where there is only one simplex that spans the cosmological distance, and it is peaked on an equilateral configuration. Further investigations could attempt a similar calculation where there was a small length scale introduced to make an elongated tetrahedron instead of an equilateral one, which would better capture the true propagator physics. Despite these caveats we still observe the characteristic repulsion of a positive cosmological constant.

\section{Summary and conclusions}

We have shown that the inclusion of a cosmological constant in a 3D Euclidean toy model of quantum gravity on a single tetrahedron reproduces the expected qualitative behavior near the infrared cutoff. Namely, we expect to have an additional repulsive force on large distance scales. The effect is reproduced in both numerical calculations and an analytic evaluation of the propagator asymptotics. This work also strengthens the argument for the interpretation of 
the cosmological constant as a deformation parameter in the theory. However, as mentioned in the introduction, we do not produce the exact form of the Newton propagator, which is thought to be due to the simplicity of the model.

This result can be taken as a first step toward performing a similar calculation in $3+1$ dimensions. Given that there are already asymptotic calculations of the propagator [4] and the $S U$ (2) symmetry is still the relevant one, this may not be too difficult for one 4-simplex. Another direction where this work could be extended is to multiple tetrahedra. The numerical computations become significantly more difficult as the number of simplicies increase, however, one could probably check the analytic results to see if the repulsion persists.

\section{Acknowledgments}

The authors would like to thank Simone Speziale and François Collet for helpful discussions. WB is funded by the Caltech Summer Undergraduate Research Fellowship Program.

\section{References}

[1] Speziale S 2006 J. High Energy Phys. 2006039

[2] Livine E R, Speziale S and Willis J L 2007 Phys. Rev. D 75024038

[3] Bonzom V, Livine E R, Smerlak M and Speziale S 2008 Nuclear Physics B 804507

[4] Bianchi E, Modesto L, Rovelli C and Speziale S 2006 Class. Quantum Grav. 236989

[5] Alesci E and Rovelli C 2013 arXiv:0711.1284v1

[6] Rovelli C and Zhang M 2011 arXiv:1105.0566

[7] Mizoguchi S and Tada T 1992 Phys. Rev. Lett. 681795

[8] Taylor Y U and Woodward C T 2003 arXiv:math/0305113

[9] Roberts J 2002 Asymptotics and 6j-symbols Geom. Topol. Monogr. 4 245-61 